\title{
Sunscreen performance of lignin from different technical resources and their general synergistic effect with synthetic sunscreens
}

Yong Qian, ", XueqingQiu ${ }^{*,+}$ and Shiping Zhu ${ }^{*,+}$

${ }^{\dagger}$ Department of Chemical Engineering, McMaster University, 1280 Main Street West, Hamilton, Ontario L8S 4L7, Canada

${ }^{\ddagger}$ School of Chemistry and Chemical Engineering, State Key Lab of Pulp and Paper

Engineering, South China University of Technology, 381 Wushan Road, Tianhe

District, Guangzhou, 510640, People's Republic of China

*Corresponding author:

Shiping Zhu

E-mail: zhuship@mcmaster.ca. Phone: +1 (905) 5259140 ext. 24962.

Xueqing Qiu

E-mail: xueqingqiu66@163.com. Phone: +86-20-87114722. Fax:+86-20-87114721. 
Table S1 Physicochemical properties of lignins from different technical resources*

\begin{tabular}{|c|c|c|c|c|c|}
\hline $\begin{array}{c}\text { Sample } \\
\text { descriptions }\end{array}$ & $\begin{array}{l}\text { Lignin, alkali } \\
\text { (AL370) }\end{array}$ & $\begin{array}{c}\text { Lignin, alkali } \\
\text { Low } \\
\text { sulfonate } \\
\text { content } \\
\text { (AL471) } \\
\end{array}$ & $\begin{array}{l}\text { Lignin, } \\
\text { Organosolv } \\
\text { (OL) }\end{array}$ & $\begin{array}{c}\text { Enzymatic } \\
\text { hydrolysis } \\
\text { lignin }^{* *} \\
(\mathrm{EHL})^{* *}\end{array}$ & $\begin{array}{l}\text { Sodium } \\
\text { lignosulfonate } \\
\text { (LS) }\end{array}$ \\
\hline \multirow{4}{*}{ Properties } & $\begin{array}{l}\text { 1. Average } \\
M_{\mathrm{w}}: 1,140\end{array}$ & $\begin{array}{l}\text { 1. Average } \\
M_{\mathrm{w}}: 10,000\end{array}$ & $\begin{array}{l}\text { 1. Average } \\
M_{\mathrm{w}}: 2,900\end{array}$ & $\begin{array}{l}\text { 1. Average } M_{\mathrm{w}} \text { : } \\
3,400\end{array}$ & $\begin{array}{l}\text { 1. Average } M_{\mathrm{w}} \text { : } \\
19,000\end{array}$ \\
\hline & $\begin{array}{l}\text { 2. Impurities: } \\
\text { 5\% moisture }\end{array}$ & $\begin{array}{l}\text { 2. Impurities: } \\
4 \% \text { sulfur }\end{array}$ & $\begin{array}{l}\text { 2. Impurities: } \\
6.5 \% \text { moisture } \\
0.2 \% \text { residual } \\
\text { sugar; } 6.6 \% \\
\text { ash; } \\
\text { Lignin } \\
\text { content: } \\
86.7 \%\end{array}$ & $\begin{array}{l}\text { 2. Impurities: } \\
\sim 5 \% \text { cellulose; } \\
\sim 15 \% \text { ash, } \\
\text { hemicellulose } \\
\text { and residual } \\
\text { enzyme; } \\
\text { Lignin } \\
\text { content }>80 \%\end{array}$ & $\begin{array}{l}\text { 2. Impurities: } \\
3.7 \% \text { sulfur } \\
\text { (organically } \\
\text { bound } \\
\text { sulfonate } \\
\text { group) }\end{array}$ \\
\hline & $\begin{array}{l}\text { 3. Functional } \\
\text { group content: } \\
\text { carboxyl } 1.39 \\
\text { mmol/g; } \\
\text { phenolic } \\
\text { hydroxyl } 2.12 \\
\text { mmol } / \mathrm{g} ; \\
\text { methoxyl } \\
5.18 \mathrm{mmol} / \mathrm{g}\end{array}$ & $\begin{array}{l}\text { 3. Functional } \\
\text { group content } \\
\text { carboxyl } \\
3.9 \% \text {; } \\
\text { phenolic } \\
\text { hydroxyl } 0.51 \\
\text { mmol/g; } \\
\text { methoxyl } \\
3.48 \mathrm{mmol} / \mathrm{g}\end{array}$ & $\begin{array}{l}\text { 3. Functional } \\
\text { group content: } \\
\text { carboxyl } 1.98 \\
\text { mmol/g; } \\
\text { phenolic } \\
\text { hydroxyl } 1.72 \\
\text { mmol/g; } \\
\text { methoxyl } \\
5.19 \mathrm{mmol} / \mathrm{g}\end{array}$ & $\begin{array}{l}3 . \quad \text { Functional } \\
\text { group content: } \\
\text { carboxyl } 1.42 \\
\text { mmol/g; } \\
\text { phenolic } \\
\text { hydroxyl } 1.14 \\
\text { mmol/g; } \\
\text { methoxyl } 2.24 \\
\text { mmol/g }\end{array}$ & $\begin{array}{l}\text { 3.Functional } \\
\text { group content: } \\
\text { carboxyl } 0.22 \\
\text { mmol/g; } \\
\text { phenolic } \\
\text { hydroxyl } 0.57 \\
\text { mmol/g } \\
\text { sulfonate group } \\
1.13 \mathrm{mmol} / \mathrm{g} ; \\
\text { methoxyl } 3.93 \\
\text { mmol/g }\end{array}$ \\
\hline & $\begin{array}{l}\text { 4. Solubility: } \\
\mathrm{H}_{2} \mathrm{O}: \\
\text { insoluble } \\
\mathrm{NaOH}: \\
\text { soluble } \\
0.05 \%\end{array}$ & $\begin{array}{l}\text { 4. Solubility } \\
\mathrm{H}_{2} \mathrm{O} \text { : soluble }\end{array}$ & $\begin{array}{l}\text { 4. Solubility: } \\
\mathrm{H}_{2} \mathrm{O} \text { : } \\
\text { insoluble } \\
\text { NaOH: } \\
\text { soluble } 0.05 \%\end{array}$ & $\begin{array}{l}\text { 4. Solubility } \\
\mathrm{H}_{2} \mathrm{O} \text { : insoluble } \\
\mathrm{NaOH} \text { : soluble } \\
0.05 \%\end{array}$ & $\begin{array}{l}\text { 4.Solubility } \\
\mathrm{H}_{2} \mathrm{O} \text { : soluble }\end{array}$ \\
\hline
\end{tabular}

*Properties of different lignin mostly come from their product instructions except the molecular weight of EHL and LS and functional group contents.

** The enzyme used for enzymatic hydrolysis is commercial cellulose enzyme Cellic CTec2, it is provided by Novozyme Co. Ltd (Tianjin, China) 
Table S2 Elemental distributions of lignin types from different technical resources.

\begin{tabular}{cccccc}
\hline Samples & $\mathrm{N} \%$ & $\mathrm{C} \%$ & $\mathrm{H} \%$ & $\mathrm{~S} \%$ & ${ }^{\circ} \%{ }^{*}$ \\
\hline AL370 & 0.52 & 62.8 & 5.74 & 1.145 & 45.985 \\
AL471 & 0.03 & 48.13 & 4.871 & 3.815 & 45.186 \\
OL & 0.65 & 60.42 & 5.445 & 0 & 33.485 \\
EHL & 1.93 & 47.51 & 5.374 & 0 & 29.795 \\
LS & 0.11 & 43.96 & 5.026 & 4.919 & 43.154 \\
\hline
\end{tabular}

*O $\%=100 \%-\mathrm{N} \%-\mathrm{C} \%-\mathrm{H} \%-\mathrm{S} \%$. 
Table S3 Active and inactive ingredients of purchased sunscreen lotion and pure cream.

\begin{tabular}{|c|c|c|}
\hline $\begin{array}{c}\text { Lotion } \\
\text { \& Cream }\end{array}$ & Active ingredients & Inactive ingredients \\
\hline LIFE SPF15 & $\begin{array}{l}\text { Octinoxate } 7.5 \% \text {, } \\
\text { Avobenzone } 4 \%\end{array}$ & $\begin{array}{l}\text { AQUA, C12-15 Alkyl benzoate, Phenethyl benzoate, Sorbitol, } \\
\text { Stearic acid, Sorbitan oleate, Benzyl alcohol, Isopropyl } \\
\text { myristate, Glyceryl stearate SE, Hydrogenated soybean oil, } \\
\text { VP/Eicosene copolymer, Triethanolamine, Imidazolidinyl } \\
\text { urea, Methylparaben, Dimethicone, Simmondsia chinensis } \\
\text { (jojoba) Seed oil, Aloe barbadensis leaf extract, Tocopherol, } \\
\text { Propylparaben, Acrylates/C10-30 Alkyl acrylate } \\
\text { crosspolymer, Carbomer, Disodium EDTA, Parfum. }\end{array}$ \\
\hline $\begin{array}{l}\text { NIVEA } \\
\text { Cream }\end{array}$ & & $\begin{array}{l}\text { Water, Glycerin, Mineral Oil, Myristyl alcohol, Butylene } \\
\text { glycol, Alcohol denat., Stearic acid, Myristyl myristate, } \\
\text { Microcrystalline wax, Glyceryl stearate, Hydrogenated } \\
\text { coco-glycerides, Simmondsia chinensis (jojoba) seed oil, } \\
\text { Tocopheryl acetate, Lanolin alcohol }\left(\text { Eucerit }^{\circledR}\right) \text {, } \\
\text { Polyglyceryl-2 caprate, Dimethicone, Carbomer, Sodium } \\
\text { hydroxide, Phenoxyethanol fragrance. }\end{array}$ \\
\hline
\end{tabular}



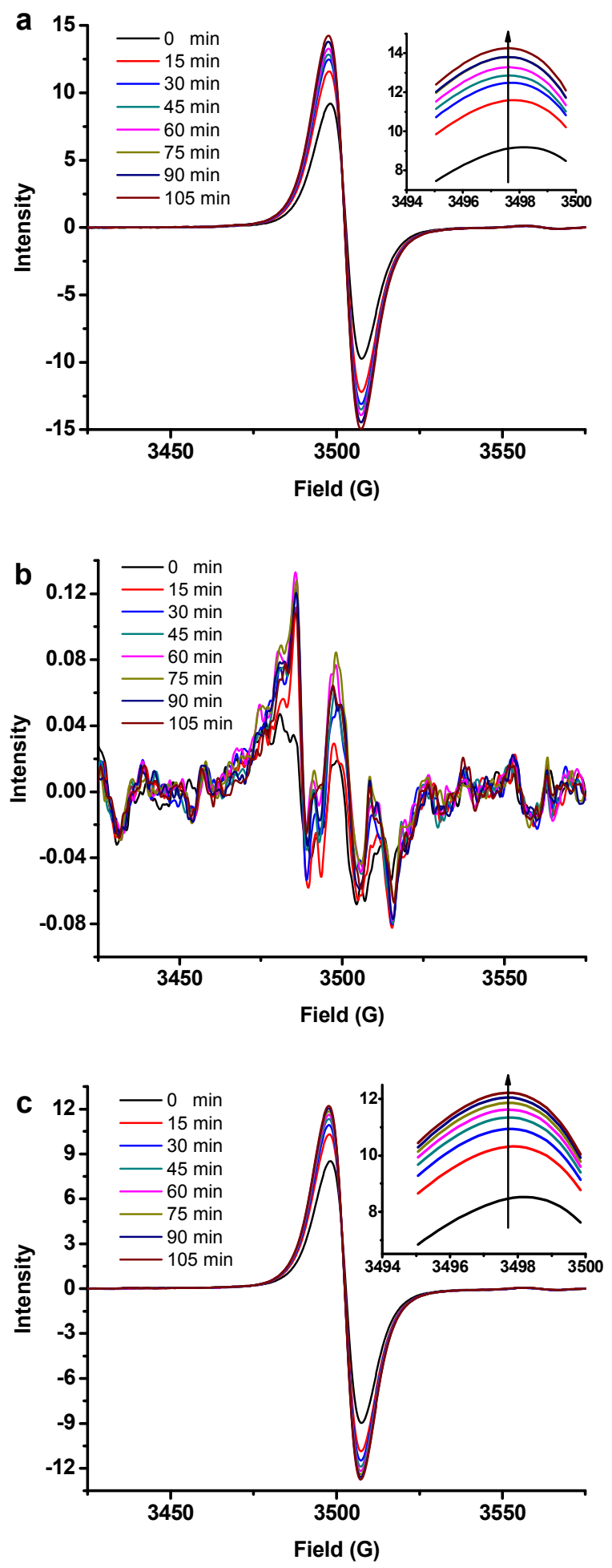

Figure S1. ESR spectra of OL (a), BMDM (b) and OL/BMDM mixture (c) under different UV radiation time. 